\title{
Revisiones por pares de protocolos presentados a un Comité Local de Investigación en Salud. Tabasco, México, 2009
}

Marco Antonio Zavala-González*

*Médico, Mgrt en Educación. Investigador del Sistema Estatal de Investigadores de Tabasco. Cárdenas. Tabasco. México.

Correspondencia: Dr. Marco Antonio Zavala González. Dirección: Calle de la alberca No. 24, Santa Rosalía, Cárdenas, Tabasco. México. Código Postal: 86470. Teléfono: +52 9371264197. Correo electrónico: zgma_51083@yahoo.com.mx

\section{RESUMEN}

Introducción: Frecuentemente se encuentran errores en protocolos y reportes de investigación, su estudio permite detectar necesidades de educación continua. Objetivo: Identificar los errores en los protocolos presentados al Comité Local de Investigación en Salud del Instituto Mexicano del Seguro Social, en Tabasco, México, durante el año 2009. Materiales y métodos: Diseño transversal descriptivo. Universo: 62 protocolos presentados en el año 2009. Muestra: no probabilística por conveniencia. Criterios de inclusión: protocolos presentados y dictaminados durante el período de estudio, que cuenten con registros de su revisión por pares. Variables: dictámenes, revisores y errores: de presentación, introducción, método y éticos. Fuente de información: archivos de revisiones por pares con base en la "Guía para la evaluación de protocolos de investigación" del Instituto Mexicano del Seguro Social. Procedimientos: sistematización de información de revisiones por pares. Análisis: estadística descriptiva. Software: Epi Info ${ }^{\circledR}$ versión 3.3.2 para entorno Windows ${ }^{\top M}$. Resultados: Se dictaminaron 50 protocolos de investigación: 32 autorizados (64\%), 10 no autorizados (20\%) y 8 con dictamen de modificarlo y volverlo a presentar (16\%). Errores más frecuentes: sintaxis y ortografía incorrectas, (62\%;), carta de consentimiento informado inadecuada, 45\%; fundamentación inadecuada del problema, 36\%; descripción insuficiente de procedimientos, 36\%. Conclusiones: Los errores en las consideraciones éticas y de redacción son los más frecuentes en los protocolos de investigación presentados al Comité Local de Investigación en Salud del Instituto Mexicano del Seguro Social en Tabasco. Se requiere capacitar en estos aspectos al personal de salud de la institución que realiza o asesora trabajos de investigación. MÉD UIS. 2015;28(3):317-25.

Palabras clave: Investigación. Manuscritos Médicos. Comités de Ética en Investigación. Revisión por Expertos. Protocolos.

\section{Peer review of research projects showed to a Health Research Local Committee. Tabasco, Mexico, 2009}

\section{ABSTRACT}

Introduction: Frequently they found fails in research projects and reports, and your study show continuing education needs. Objective: to identify errors in projects presented to Health Research Local Committee of the Instituto Mexicano del Seguro Social from Tabasco, Mexico, during 2009. Material and methods: Cross-sectional and descriptive design. Universe: 62 projects presented in 2009. Sample: no randomized for convenience. Inclusion criteria: projects presented and ruled in 2009 that and counted with peer review files. Variables: verdicts, reviewers, presentation errors, introduction errors, method errors, ethic methods. Information source: peer review files based on "Research projects evaluation guide" of the Instituto Mexicano del Seguro Social. Procedures: systematization of peer review information. Analysis: descriptive statistic. Software: Epi Info® version 3.3.2 for Windows ${ }^{\mathrm{TM}}$ environment. Results: 50 research projects were ruled: 32 authorized (64\%), 10 non-authorized (20\%), 8 with modification and sending back to peer-review (16\%). As the most frecuent errors, were found: incorrect syntax and spelling $62 \%$, inadequate informed consent letter $45 \%$, lack of appropriate foundation $36 \%$, insufficient description of procedures $36 \%$. Conclusions: The most frequents errors in the research projects presented to the Health Research Local Committee of the Instituto Mexicano del Seguro Social were about the ethical considerations and the redaction. For this reason to qualify in these aspects to health personnel of institution that carry out and advice on research works is required. MÉD UIS. 2015;28(3):317-25.

Keywords: Research. Manuscripts, Medical. Ethics Committees. Research. Peer Review. Protocols

Artículo recibido el 21 de abril de 2015 y aceptado para publicación el 25 de septiembre de 2015 . 
¿Cómo citar este artículo?: Zavala-González MA. Revisiones por pares de protocolos presentados a un Comité Local de Investigación en Salud. Tabasco, México, 2009. MÉD UIS. 2015;28(3):317-25.

\section{INTRODUCCIÓN}

El médico se desarrolla principalmente en el área asistencial, unos pocos en docencia y en menor medida en investigación. Los ejes de la formación médica incluyen el adquirir conocimientos y la capacidad para generarlos; aunque lo ideal sería que todos los médicos fueran capaces de desarrollarlos a través de la docencia e investigación, pocos lo pueden hacer'. Algunos autores coinciden en que uno de los factores que más contribuye a la génesis de la escasa producción científica en Latinoamérica, es la deficiente formación en el área durante el pregrado ${ }^{2-5}$, que suele prevalecer durante los cursos de especialización, viéndose apenas afectada con la formación de maestros y doctores ${ }^{6}$. No obstante, la práctica diaria requiere la toma de decisiones sobre actividades preventivas, diagnósticas, terapéuticas y de pronóstico basadas en probabilidades que pretenden delimitar la incertidumbre que envuelve a la medicina ${ }^{7}$. Con frecuencia, existen dificultades para trasladar los resultados de una investigación a la práctica clínica por la forma en que habitualmente se presentan los resultados, en términos de $p$ $<0,05, p<0,01$, riesgo relativo, odds ratio, reducción absoluta del riesgo, reducción relativa del riesgo, fracción atribuible poblacional o fracción etiológica, entre otros. A este lenguaje con el que muchos profesionales sanitarios no están familiarizados y tienen dificultades para su comprensión, se suma el hecho que, los resultados de una investigación pueden ser estadísticamente significativos y no clínicamente relevantes ${ }^{8-10}$.

Dentro de este contexto diversos autores se han dado a la tarea de analizar los errores observados con mayor frecuencia en, resúmenes de trabajos de investigación sometidos a revisión para su presentación en foros y congresos, reportes de trabajos de investigación observacionales y experimentales, e incluso, en traducciones de artículos del inglés al español. Como resultado, se ha informado que los errores más frecuentes son manipulación de datos, errores en los criterios de selección, análisis incorrecto de observaciones múltiples y fabricación de datos, entre otros expuestos en la Tabla $1^{11-25}$. Esta situación de errores metodológicos y estructurales no solo es presentada por las investigaciones de corte cuantitativo en ciencias de la salud, sino que también ha sido referida en investigaciones cualitativas, de uso principal en ciencias sociales ${ }^{26,27}$.

Tabla 1. Errores más frecuentes en resúmenes y reportes de investigación

Error aleatorio
No reporte del método de asignación de la intervención
Poder estadístico inadecuado
Falta de apego al tratamiento
Clasificación errónea del resultado en estudio
Falsificación de datos
Manipulación de datos
Errores en la recolección activa de datos
Errores en los criterios de selección
Análisis incorrecto de observaciones múltiples
Contaminación del estudio

Seguimiento insuficiente o prolongado
Seguimiento sesgado
Plagio
Método inadecuado de generación de números aleatorios
Método incorrecto de comparación de subgrupos
Sobre-utilización del análisis de intención de tratar
Competencia de riesgo
Fabricación de datos
Robo de datos
Inflar la hoja de vida

Fuente: Elaboración propia con base en información obtenida de la literatura consultada ${ }^{11-25}$.

Los errores y sesgos en los reportes de trabajos de investigación analizados posteriormente para su publicación, por editores, revisores, críticos y estudiosos de la metodología de la investigación, pueden ser atribuidos a los dictámenes en su fase de protocolo, cuando son sometidos a evaluación por el
Comité Local de Investigación en Salud (CLIS), que vela por la calidad de las investigaciones y el cumplimiento de las normas éticas en una institución, en tanto que este organismo es quien dictamina la aprobación o rechazo de las investigaciones propuestas; de tal modo, que un reporte deficiente, es consecuencia de un 
protocolo deficiente ${ }^{28-31}$. La aceptación condicionada o rechazo de proyectos, genera inconformidad y quejas en quienes reciben el dictamen. No obstante, aceptar sin condiciones proyectos que presentan deficiencias metodológicas y éticas, por mera política, tiene una repercusión directa sobre la calidad y cantidad de las publicaciones.

EI CLIS 2701 del Instituto Mexicano del Seguro Social (IMSS), es un organismo multidisciplinario que tiene la tarea de velar por la calidad de las investigaciones realizadas en el IMSS, Delegación Tabasco, y de que se cumplan las normas éticas nacionales e internacionales en los mismos. Este comité se ubica en el Hospital General de Zona No. 2 de la referida institución, un hospital de segundo nivel que se desempeña como centro de referencia para las todas las unidades médicas de primer nivel de la región Chontalpa del estado de Tabasco. En el CLIS 2701 del IMSS, en Tabasco, México, todos los protocolos recibidos, corresponden a investigaciones realizadas por becarios para liberar ciclos clínicos, internado y servicio social, así como para la obtención de diplomas de especialidad médica, asesoradas por personal cualificado de la institución. Identificar los errores cometidos en los proyectos presentados al CLIS, permitiría identificar necesidades de educación continua, que al ser satisfechas, se traducirían en un menor número de proyectos rechazados y condicionados, generando una mayor proporción de publicación de dichos trabajos de investigación.

Por consiguiente, se realizó la presente investigación con el objetivo de caracterizar los errores en los protocolos presentados al CLIS 2701 del IMSS, en Tabasco, México, durante el año 2009, mediante la sistematización y el análisis de la información de las revisiones por pares.

\section{Materiales y Métodos}

Se realizó una investigación con metodología cuantitativa, empleando un diseño epidemiológico transversal descriptivo ${ }^{32}$. El universo de estudio estuvo constituido por 62 protocolos correspondientes a investigaciones realizadas por becarios con finalidad académica presentados al CLIS 2701 del IMSS, durante el período de enero a diciembre de 2009. Se estudió una muestra no probabilística por conveniencia, en la que se incluyeron todos los protocolos que cumplieron con los criterios de selección definidos: haber sido presentados y dictaminados durante el año de estudio y contar con registros de su revisión por el CLIS. Se excluyeron los protocolos presentados durante el mismo período que no recibieron dictamen en el mismo año o no contaron con registros de su revisión por pares.

Se las estudiaron variables: dictamen, revisores, errores en la presentación, errores en la introducción, método y consideraciones éticas; cuyas definiciones y parámetros de medición se exponen en la Tabla 2. El patrón de referencia para su estudio fue la "Guía para la evaluación de protocolos de investigación” del IMSS33, que cada uno de los expertos remitió completamente diligenciado por cada protocolo evaluado.

Se solicitó al CLIS 2701 del IMSS los archivos de los protocolos remitidos a este para su evaluación durante el período; se identificaron las enmiendas solicitadas y los motivos de rechazo, de acuerdo a la Guía para la evaluación de protocolos de investigación. Se solicitaron copias de los documentos referidos y con base en las variables evaluadas, se manufacturó una matriz para la sistematización de la información utilizando el software Epi Info ${ }^{\circledR}$ versión 3.3 .2 (freeware distribuido por el Center of Disease Control and Prevention de E.U.A., a través de www.cdc.gov), mediante el cual se realizó el análisis descriptivo univariado a través de frecuencias absolutas y relativas.

Con base en el Artículo 17 del Reglamento de la Ley General de Salud de México, en materia de investigación para la salud ${ }^{34}$, la presente investigación fue clasificada como "sin riesgo", en virtud de que empleó técnicas y métodos de investigación documental retrospectivos, en los que no se identificaron ni trataron aspectos sensitivos de la conducta de los investigadores, por lo que no se requirió consentimiento informado.

\section{Resultados}

Se revisaron 62 protocolos de los cuales, tres presentaron solicitud de cancelación $(4,8 \%)$ y nueve fueron dictaminados en el 2010 (14,5\%), de modo que se estudiaron 50 protocolos ( $80,7 \%$ del universo). El número de revisores por protocolo fue dos en todos los casos. Se encontraron 32 (64\%) protocolos autorizados, 10 (20\%) no autorizados y ocho (16\%) con dictamen de modificarlo y volverlo a presentar. En cuanto a los errores en la presentación, lo más frecuente fue la sintaxis y la ortografía incorrectas, presente en todos los protocolos de investigación no autorizados y con dictamen de modificar el 
proyecto y volverlo a presentar, e inclusive, en los protocolos que recibieron dictamen de autorizado (Ver Tabla 3).

Respecto a los errores más frecuentes en la introducción fueron los referentes al planteamiento del problema, en donde fue común observar una inadecuada fundamentación de este; la falta de aislamiento del problema aludido de entre otros similares y la ausencia de identificación de variables o la relación entre ellas, errores que se presentaron en proporción similar tanto en los protocolos no autorizados como en aquellos con dictamen de modificar y volver a presentar. Se observó también, que entre los protocolos de investigación autorizados, en todos los casos, hay registro de errores relacionados con la formulación de las hipótesis, lo que de acuerdo a los comentarios de los revisores, es atribuible a que los trabajos de investigación autorizados no tenían hipótesis por no ser pertinente su formulación dado el diseño del estudio. Por otra parte, no se encontró que la información de los antecedentes no se relacionara directamente con el problema, que se fundamentaran inadecuadamente los objetivos, ni que las referencias bibliográficas estuvieran mal señaladas o no concordaran con las afirmaciones atribuidas (Ver Tabla 4).

Tabla 2. Definición de variables

\begin{tabular}{|c|c|c|}
\hline Variables & Definiciones & $\begin{array}{l}\text { Parámetros de } \\
\text { medición }\end{array}$ \\
\hline Dictamen & $\begin{array}{l}\text { Resultado de la evaluación del protocolo por parte } \\
\text { del CLIS }\end{array}$ & $\begin{array}{l}\text { No autorizado, } \\
\text { modificar proyecto, } \\
\text { autorizado }\end{array}$ \\
\hline Revisores & $\begin{array}{l}\text { Número de expertos académicos que evaluaron el } \\
\text { protocolo para el CLIS }\end{array}$ & Número de revisores \\
\hline \multirow{2}{*}{ Errores en la presentación } & Errores de sintaxis y ortografía & Ausente, presente \\
\hline & Errores de otorgamiento de créditos & Ausente, presente \\
\hline \multirow{6}{*}{ Errores en la introducción } & Errores en el título & Ausente, presente \\
\hline & Errores en los antecedentes científicos & Ausente, presente \\
\hline & Errores en el planteamiento del problema & Ausente, presente \\
\hline & Errores en las referencias bibliográficas & Ausente, presente \\
\hline & Errores en los objetivos & Ausente, presente \\
\hline & Errores en las hipótesis & Ausente, presente \\
\hline \multirow{11}{*}{ Errores en el método } & Errores en el universo y la muestra & Ausente, presente \\
\hline & Errores en los criterios de selección & Ausente, presente \\
\hline & Errores en la definición de variables & Ausente, presente \\
\hline & Ausencia de reproducibilidad & Ausente, presente \\
\hline & Mala descripción del estudio & Ausente, presente \\
\hline & Mala descripción de la sistematización & Ausente, presente \\
\hline & Ausencia de control de calidad & Ausente, presente \\
\hline & Descripción inadecuada del plan de análisis & Ausente, presente \\
\hline & Errores de congruencia objetivo-diseño & Ausente, presente \\
\hline & Falta de originalidad & Ausente, presente \\
\hline & Falta de trascendencia & Ausente, presente \\
\hline \multirow{4}{*}{ Errores en las consideraciones éticas } & Ausencia de consentimiento informado & Ausente, presente \\
\hline & Consentimiento informado inadecuado & Ausente, presente \\
\hline & Lenguaje inaccesible del consentimiento & Ausente, presente \\
\hline & Selección parcial o sesgada de los sujetos & Ausente, presente \\
\hline
\end{tabular}


Tabla 3. Errores en la presentación

\begin{tabular}{|c|c|c|c|c|c|c|c|c|}
\hline \multirow{3}{*}{ Error } & \multicolumn{6}{|c|}{ Dictamen } & & \\
\hline & \multicolumn{2}{|c|}{$\begin{array}{c}\text { No } \\
\text { autorizado } \\
(n=10)\end{array}$} & \multicolumn{2}{|c|}{$\begin{array}{c}\text { Modificar } \\
\text { proyecto } \\
(n=8)\end{array}$} & \multicolumn{2}{|c|}{$\begin{array}{c}\text { Autorizado } \\
(n=32)\end{array}$} & \multicolumn{2}{|c|}{$\begin{array}{c}\text { Total } \\
(n=50)\end{array}$} \\
\hline & $\mathbf{F}$ & $\%$ & $\mathbf{F}$ & $\%$ & $\mathbf{F}$ & $\%$ & $\mathbf{F}$ & $\%$ \\
\hline La sintaxis y la ortografía son incorrectas & 10 & 100 & 8 & 100 & 13 & 40.6 & 31 & 62 \\
\hline Otorga los créditos en forma inadecuada & 10 & 100 & 8 & 100 & 0 & 0 & 18 & 36 \\
\hline
\end{tabular}

Nota: La tabla no ofrece la sumatoria de resultados por columna ni fila, dado que se evaluó individualmente la frecuencia de presentación de cada error sin considerar su ausencia

Fuente: Autores.

\section{Tabla 4. Errores en la introducción}

\begin{tabular}{|c|c|c|c|c|c|c|c|c|}
\hline \multirow{3}{*}{ Error } & \multicolumn{6}{|c|}{ Dictamen } & \multirow{2}{*}{\multicolumn{2}{|c|}{$\begin{array}{c}\text { Total } \\
(n=50)\end{array}$}} \\
\hline & \multicolumn{2}{|c|}{$\begin{array}{l}\text { No autorizado } \\
\qquad(n=10)\end{array}$} & \multicolumn{2}{|c|}{$\begin{array}{l}\text { Modificar } \\
\text { proyecto } \\
(n=8)\end{array}$} & \multicolumn{2}{|c|}{$\begin{array}{l}\text { Autorizado } \\
\qquad(\mathrm{n}=32)\end{array}$} & & \\
\hline & $\mathbf{F}$ & $\%$ & $\mathbf{F}$ & $\%$ & $F$ & $\%$ & $\mathbf{F}$ & $\%$ \\
\hline $\begin{array}{l}\text { El título no se ajusta a las características del } \\
\text { trabajo }\end{array}$ & 2 & 20 & 2 & 25 & 1 & 3.10 & 5 & 10 \\
\hline $\begin{array}{l}\text { Se fundamenta de manera inadecuada el } \\
\text { planteamiento }\end{array}$ & 10 & 100 & 8 & 100 & 0 & 0 & 18 & 36 \\
\hline $\begin{array}{l}\text { Se fundamentan de manera inadecuada los } \\
\text { métodos }\end{array}$ & 10 & 100 & 8 & 100 & 0 & 0 & 18 & 36 \\
\hline $\begin{array}{l}\text { No identifica claramente el problema ni lo aísla } \\
\text { de otros similares }\end{array}$ & 10 & 100 & 8 & 100 & 0 & 0 & 18 & 36 \\
\hline $\begin{array}{l}\text { En el planteamiento del problema no se } \\
\text { identifican las variables en estudio o la relación } \\
\text { entre ellas }\end{array}$ & 10 & 100 & 0 & 0 & 0 & 0 & 10 & 20 \\
\hline $\begin{array}{l}\text { El objetivo general está planteado de manera } \\
\text { que no permite diseñar un estudio para lograr su } \\
\text { consecución }\end{array}$ & 10 & 100 & 0 & 0 & 0 & 0 & 10 & 20 \\
\hline $\begin{array}{l}\text { Cada objetivo específico está planteado de } \\
\text { manera que no permite diseñar un estudio para } \\
\text { lograr su consecución }\end{array}$ & 10 & 100 & 0 & 0 & 0 & 0 & 10 & 20 \\
\hline $\begin{array}{l}\text { El objetivo general está inadecuadamente } \\
\text { operacionalizado }\end{array}$ & 10 & 100 & 8 & 100 & 0 & 0 & 18 & 36 \\
\hline $\begin{array}{l}\text { Uno o más de los objetivos específicos está } \\
\text { inadecuadamente operacionalizado }\end{array}$ & 10 & 100 & 8 & 100 & 0 & 0 & 18 & 36 \\
\hline $\begin{array}{l}\text { No hay un objetivo por cada hipótesis que se } \\
\text { planteó }\end{array}$ & 10 & 100 & 8 & 100 & 0 & 0 & 18 & 36 \\
\hline $\begin{array}{l}\text { Las hipótesis no predicen lógicamente la } \\
\text { respuesta probable a las preguntas que se } \\
\text { formularon en el planteamiento del problema }\end{array}$ & 10 & 100 & 8 & 100 & 32 & 100 & 50 & 100 \\
\hline $\begin{array}{l}\text { No se identifica la dirección y el compromiso de } \\
\text { cada hipótesis }\end{array}$ & 10 & 100 & 8 & 100 & 32 & 100 & 50 & 100 \\
\hline
\end{tabular}

Nota: La tabla no ofrece la sumatoria de resultados por columna ni fila, dado que se evaluó individualmente la frecuencia de presentación de cada error sin considerar su ausencia

Fuente: Autores. 
Los errores más frecuentes en el método fueron la definición de variables, la reproducibilidad y validez de los métodos e instrumentos de medición, la descripción de los procedimientos, y en general, la sistematización de la información para llevar a cabo el estudio. Se encontró una pequeña proporción de errores de método entre los protocolos de investigación autorizados, referentes a la caracterización del universo, el control de calidad de la información, e incongruencia entre los elementos teóricos y metodológicos del protocolo de investigación, los cuales, no afectaron el dictamen de los protocolos en cuestión. Asimismo, no se encontró que no se describieran los criterios de selección, que los criterios de selección fueran inadecuados para el estudio, que el trabajo no aportara algo nuevo a nivel nacional, ni que el trabajo no fuera importante de acuerdo a su aportación, avance del conocimiento o aplicabilidad (Ver Tabla 5).

Tabla 5. Errores en el método

\begin{tabular}{|c|c|c|c|c|c|c|c|c|}
\hline \multirow{3}{*}{ Error } & \multicolumn{6}{|c|}{ Dictamen } & \multirow{2}{*}{\multicolumn{2}{|c|}{$\begin{array}{c}\text { Total } \\
(n=50)\end{array}$}} \\
\hline & \multicolumn{2}{|c|}{$\begin{array}{l}\text { No autorizado } \\
\qquad(n=10)\end{array}$} & \multicolumn{2}{|c|}{$\begin{array}{l}\text { Modificar } \\
\text { proyecto } \\
(\mathbf{n}=\mathbf{8})\end{array}$} & \multicolumn{2}{|c|}{$\begin{array}{l}\text { Autorizado } \\
\qquad(n=32)\end{array}$} & & \\
\hline & $F$ & $\%$ & $F$ & $\%$ & $F$ & $\%$ & $F$ & $\%$ \\
\hline $\begin{array}{l}\text { Está mal caracterizado el universo de trabajo y la } \\
\text { propuesta para obtener la muestra necesaria y } \\
\text { adecuada para el estudio }\end{array}$ & 5 & 50 & 2 & 25 & 1 & 3.10 & 8 & 16 \\
\hline $\begin{array}{l}\text { La definición de las variables, tanto la conceptual } \\
\text { como la operacional, es confusa y/o inadecuada }\end{array}$ & 10 & 100 & 8 & 100 & 0 & 0 & 18 & 36 \\
\hline $\begin{array}{l}\text { La reproducibilidad y validez de los métodos y/o } \\
\text { instrumentos de medición que se utilizarán en el } \\
\text { estudio no está descrita y/o es inadecuada }\end{array}$ & 10 & 100 & 8 & 100 & 0 & 0 & 18 & 36 \\
\hline $\begin{array}{l}\text { La descripción del estudio es confusa y/o está } \\
\text { insuficientemente detallada }\end{array}$ & 10 & 100 & 8 & 100 & 0 & 0 & 18 & 36 \\
\hline $\begin{array}{l}\text { La sistematización de la recolección de los datos } \\
\text { es inadecuada }\end{array}$ & 10 & 100 & 8 & 100 & 0 & 0 & 18 & 36 \\
\hline $\begin{array}{l}\text { La descripción de los procedimientos, tanto } \\
\text { observacionales como experimentales, es } \\
\text { confusa y/o no está insuficientemente detallada }\end{array}$ & 10 & 100 & 8 & 100 & 0 & 0 & 18 & 36 \\
\hline $\begin{array}{l}\text { El estudio no incluye alguna forma de control de } \\
\text { calidad }\end{array}$ & 10 & 100 & 8 & 100 & 1 & 3 & 19 & 38 \\
\hline $\begin{array}{l}\text { No se describen los métodos para procesar los } \\
\text { datos ni la propuesta para el análisis estadístico } \\
\text { que se planea utilizar }\end{array}$ & 0 & 0 & 0 & 0 & 0 & 0 & 0 & 0 \\
\hline $\begin{array}{l}\text { El análisis estadístico que se propone no es } \\
\text { acorde a la forma en que se calculó el tamaño de } \\
\text { la muestra }\end{array}$ & 10 & 100 & 8 & 100 & 0 & 0 & 18 & 36 \\
\hline $\begin{array}{l}\text { Existe incongruencia entre la pregunta, el diseño } \\
\text { y la descripción del estudio }\end{array}$ & 4 & 40 & 2 & 25 & 1 & 3 & 7 & 14 \\
\hline $\begin{array}{l}\text { Los objetivos y la metodología no permitirán } \\
\text { contestar la pregunta planteada }\end{array}$ & 10 & 100 & 4 & 50 & 0 & 0 & 14 & 28 \\
\hline No aporta algo nuevo a nivel internacional & 6 & 60 & 7 & 88 & 0 & 0 & 13 & 26 \\
\hline
\end{tabular}

Nota: La tabla no ofrece la sumatoria de resultados por columna ni fila, dado que se evaluó individualmente la frecuencia de presentación de cada error sin considerar su ausencia.

Fuente: Autores. 
Finalmente, en lo que se refiere a los errores en las consideraciones éticas, estos sólo fueron examinados en los protocolos que por la naturaleza del estudio debían de diligenciar carta de consentimiento informado, de modo que se excluyeron de este análisis 10 protocolos autorizados que no la solicitaron. Así pues, en lo concerniente a este tipo de errores, se encontró que los más frecuentes fueron la carta de consentimiento informado inadecuada o incompleta, lenguaje inaccesible para los pacientes, manipulación de la decisión del paciente, e inadecuada descripción de riesgos y beneficios tanto para los pacientes como para la institución. Sin embargo, no se observó que no se presentara la carta de consentimiento informado, ni que la selección de los sujetos de estudio fuera parcial, con sesgo social, racial, sexual o cultural (Ver Tabla 6).

Tabla 6. Errores en las consideraciones éticas

\begin{tabular}{|c|c|c|c|c|c|c|c|c|}
\hline \multirow{3}{*}{ Error } & \multicolumn{6}{|c|}{ Dictamen } & & \\
\hline & \multicolumn{2}{|c|}{$\begin{array}{l}\text { No autorizado } \\
\qquad(n=10)\end{array}$} & \multicolumn{2}{|c|}{$\begin{array}{l}\text { Modificar } \\
\text { proyecto } \\
(\mathbf{n}=\mathbf{8})\end{array}$} & \multicolumn{2}{|c|}{$\begin{array}{l}\text { Autorizado } \\
\qquad(\mathrm{n}=22)\end{array}$} & \multicolumn{2}{|c|}{$\begin{array}{l}\text { Total } \\
(n=40)\end{array}$} \\
\hline & $F$ & $\%$ & $F$ & $\%$ & $F$ & $\%$ & $\mathbf{F}$ & $\%$ \\
\hline $\begin{array}{l}\text { La carta de consentimiento informado es } \\
\text { inadecuada e incompleta para el estudio }\end{array}$ & 10 & 100 & 8 & 100 & 0 & 0 & 18 & 45 \\
\hline $\begin{array}{l}\text { El lenguaje de la carta de consentimiento } \\
\text { informado es inaccesible para el paciente }\end{array}$ & 10 & 100 & 7 & 88 & 1 & 5 & 18 & 45 \\
\hline $\begin{array}{l}\text { La redacción de la carta de consentimiento } \\
\text { informado manipula la decisión del paciente }\end{array}$ & 10 & 100 & 8 & 100 & 0 & 0 & 18 & 45 \\
\hline $\begin{array}{l}\text { En la carta no se describen con claridad los } \\
\text { riesgos y beneficios para los participantes }\end{array}$ & 10 & 100 & 8 & 100 & 0 & 0 & 18 & 45 \\
\hline $\begin{array}{l}\text { El protocolo no considera una adecuada } \\
\text { evaluación de los riesgos y beneficios de su } \\
\text { realización }\end{array}$ & 10 & 100 & 8 & 100 & 0 & 0 & 18 & 45 \\
\hline
\end{tabular}

Fuente: Autores.

\section{Discusión}

Respecto a los resultados obtenidos, cabe resaltar el hecho de que esta es la primera investigación conocida por el autor al momento del estudio en la que se exploran los errores presentes en los protocolos de investigación, dado que la literatura consultada durante este periodo informa estos errores en otro tipo de productos científicos, tales como resúmenes y reportes de investigación ${ }^{11-25}$, así como traducciones de éstos, cuya estructura, extensión y contenidos son diferentes; de modo que los resultados observados en esta serie no son comparables con los referidos en la citada literatura, desconociendo si actualmente existen investigaciones más recientes en las que se haya evaluado el mismo tipo de documentos científicos valorados en este trabajo.

Se encontró que los errores más frecuentemente reportados por revisores en protocolos de investigación son: sintaxis y ortografía incorrecta, carta de consentimiento informado mal dirigida, fundamentación inadecuada del problema y descripción insuficiente de procedimientos, entre otros de corte similar; lo que concuerda en parte con lo reportado por autores como Schroter ${ }^{11}$, Douglas ${ }^{12}$, Persell ${ }^{15}$, Strazak ${ }^{20}$ y Ségalat ${ }^{21}$, quienes han reportado como principales errores de reportes de trabajos de investigación publicados, la descripción errónea o insuficiente del método y los procedimientos relativos a la investigación, hecho que imposibilita la adecuada comprensión del estudio, y con ello, la reproducibilidad del mismo.

Al mismo tiempo, los errores cometidos respecto a las consideraciones éticas, concuerdan sustancialmente con lo aludido por Reyes ${ }^{14}$, quien señala que el error ético más frecuentemente cometido es el uso de lenguaje técnico en el consentimiento informado, con lo que la información proporcionada por este queda sujeta a la subjetividad del investigador, manipulando la decisión del paciente en cuanto a su participación. Mientras que el resto de los errores en el método y las consideraciones éticas referidos en 
la literatura consultada ${ }^{11-25}$, no fueron encontrados en esta serie, en virtud que se trató de protocolos y no de reportes de investigación; no obstante, hay que tener presente que un reporte de investigación deficiente, es necesariamente consecuencia de un protocolo deficiente, como lo refieren Rakestraw ${ }^{28}$, Robinson ${ }^{29}$, Sengupta ${ }^{30}$ y Silbergeld ${ }^{31}$ en diferentes momentos, latitudes y contextos.

Es menester comentar de igual forma las limitaciones y sesgos potenciales de la presente investigación, en este sentido, la principal limitación de este estudio es que sus resultados corresponden a un único escenario y población con características académicas, idiosincrásicas y sociodemográficas particulares, de modo que no pueden ser generalizables para lo que se requeriría de un estudio multicéntrico, por lo que los hallazgos sólo tienen utilidad práctica en el mismo contexto, quedando en calidad de referente para futuros estudios en otras latitudes. Por otro lado, respecto a los sesgos, si bien se incluyeron a todos los sujetos susceptibles de ser incluidos, minimizando los errores tipo I y II, existe un potencial error de observación dado que la recolección de datos fue retrospectiva, de modo que la calidad de la información con la que se trabajó la definición conceptual y operacional de las variables escapó al control del investigador, lo que corresponde a un sesgo inherente a los estudios de corte retrospectivo.

\section{Conclusiones}

Existe una considerable cantidad de errores de toda índole en los protocolos de investigación presentados al CLIS 2701 del IMSS, Delegación Tabasco, incluso en protocolos con dictamen de autorizado, entre los que, los errores en las consideraciones éticas y de redacción eran los más frecuentes en los protocolos de investigación presentados al comité, lo que denota la importancia del proceso de revisión por pares en la evaluación de los protocolos de investigación, especialmente en cuanto a cuestiones éticas se refiere. Estos resultados evidencian la necesidad de proporcionar educación continua al personal de salud que realiza y asesora trabajos de investigación en este ámbito dentro de la institución, en materia de redacción de textos científicos, métodos de investigación y aspectos éticos de la investigación en seres humanos, para lo que se sugiere utilizar el método de curso-taller, de modo que se logre mejorar la calidad de los protocolos y reportes realizados, hecho que podría incidir favorablemente sobre el indicador institucional referente a la proporción de trabajos de investigación aprobados cuyos resultados son publicados en revistas arbitradas o indexadas, misma que se sugiere explorar en futuros estudios. Asimismo, se sugiere realizar estudios cuasi-experimentales en relación a las intervenciones que se propongan o desarrollen para la solución de este problema, en aras de documentar su impacto y difundir los casos de éxito para reproducirlos en otros contextos.

\section{AgradeCIMientos}

El autor agradece a la Dra. María Lilia Ascencio Barreda y al Dr. Rafael Antonio Vivas Pérez, del Instituto Mexicano del Seguro Social, Delegación Tabasco, las facilidades otorgadas para la realización de este estudio, y a los Drs. Juan Manuel Junco Carrera y Sergio Eduardo Posada Arévalo, de la Universidad Virtual en Ciencias de la Salud y de la Universidad Juárez Autónoma de Tabasco, respectivamente, su valiosa contribución en la revisión del manuscrito.

\section{Financiamiento}

Trabajo realizado con recursos del autor, sin financiamiento ni apoyo material de ninguna otra índole por parte de instituciones u organismos públicos o privados.

\section{Conflicto de InTERÉS}

El presente trabajo no presenta conflictos de intereses económicos, institucionales o personales.

\section{INFORMACIÓN ADICIONAL}

Trabajo realizado por el autor, para obtener el Diploma en Investigación en Ciencias de la Salud con Tecnología Digital, expedido por la Facultad de Estudios Superiores Iztacala de la Universidad Nacional Autónoma de México.

\section{ReFERENCIAS BiblogeráficAS}

1. Amato D, de Jesús Novales-Castro X. Feasibility of implementing a learning based problem solving and peer evaluation approach among medical students in Mexico. Gac Med Mex 2009;145(3):197-205.

2. Falconí E. La responsabilidad del Estado y las instituciones académicas en la investigación en salud pública. Rev Peru Med Exp Salud Publica 2007;24(4):440-1.

3. Molina-Ordóñez J, Huamaní C, Mayta-Tristán P. Apreciación estudiantil sobre la capacitación universitaria en investigación: 
Estudio preliminar. Rev Peru Med Exp Salud Publica 2008;25(3):325-9.

4. Díaz-Vélez C, Manrique-González LM, Galán-Rodas E, ApolayaSegura M. Conocimientos, actitudes y prácticas de investigación de los estudiantes de pregrado de Facultades de Medicina del Perú. Acta Med Per 2008;25(1):9-15.

5. Arroyo-Hernández H, De la Cruz W, Miranda-Soberon UE. Dificultades para el desarrollo de investigaciones en pregrado en una universidad pública de provincia, Perú. Rev Peru Med Exp Salud Publica 2008;25(4):448-9.

6. Enserink M. Scientific Publishing. Elseiver to editor: change controversial journal or resign. Science 2010;327(5971):1316.

7. Coll G, De la Figuera M, Vinyoles E, Albaladejo C, Dalfó A. Las dificultades de la investigación en atención primaria: A propósito de un estudio. Butlletí [serie en internet] 2009;27:9 [consultado, julio 2014]. Disponible en http://butlleti.camfic.org/Volum 26/ AE_Dificultades_Investigacio_Estudi_CAST.aspx

8. Hauser SL, Johnston SC, Ferriero DM, Lowestein DH, Josephson SA, Messing RO, et al. Status report: the Annals in 2010. Ann Neurol. 2010;67(1):A5-12.

9. Platt JA. Remembering the past, an eye to the future. Oper Dent. 2010;35(1):1-2.

10. Liesegan TJ. Peer review should continue after publication. Am J Ophthalmol. 2010;149(3):359-60.

11. Schroter S, Black N, Evans S, Godlee F, Osorio L, Smith R. What errors do peer reviewers detect, and does training improve their ability to detect them?. J R Soc Med.2008;101:507-14.

12. Douglas G, Altman DSc. Poor-quality medical research. What Can Journals Do? JAMA.2005;287(21):2765-7.

13. Anaya R, Grover F, Centeno N, Godínez M. Ensayos clínicos sin significado estadístico. La importancia del error tipo II. Cirugía y Cirujanos 2008;76(3):271-5.

14. Reyes H, Palma J, Andresen M. Ethics in articles published in medical journals. 2007;135(4):529-33

15. Persell SD, Dolan NC, Friesema EM, Thompson JA, Kaiser D, Baker DW. Frequency of inappropiate medical exceptions to quality measures. Ann Intern Med 2010;152(4):225-31.

16. Jung BC. Conflicts of interest, authorship, and disclousers in industry-related scientific publications. Mayo Clin Proc 2010;85(2):199.

17. Scientific fraud: action needed in China. Lancet 2010;375(9709):94

18. Conclusion by exclusión. Nat Genet 2010;42(2):95.

19. Fernández P. Investigación en atención primaria. Cad Aten Primaria 2005;12:41-4.

20. Strazak A, Zarman Q, Pfeiffer K, Göbel G, Ulmer H. Statistical errors in medical research - a review of common pitfalls. Swiss Med Wkly 2007;137:44-9.

21. Ségalat L. System crash. EMBO 2010;11(2):86-9.

22. Texidor-Pellón R, Reyes-Miranda D. Algunas reflexiones acerca de los errores más frecuentes encontrados en textos médicos traducidos del idioma inglés. Educ Med Superior 2009;23(4):220-5.

23. Tseng I. General questions about Peer Review. J Mich Den Assoc 2009;91(12):20.

24. Nofziger AC, Naumburg EH, Davis BJ, Mooney CJ, Epstein RM. Impact to peer assessment on the professional development of medical students: a qualitative study. Acad Med 2010;85(1):140-7.

25. Freedman R. Learning to review. J Clin Psychiatry 2009:70(11):1599-600.

26. Wolff S, Robles F. Diez errores y máximas en la investigación social cualitativa. Articulando metodológicas comunes entre etnometodología y la teoría de sistemas. Sociedad Hoy 2006;11:111-25.

27. Bhagianadh D, Banderkar S, Ravindran D, Nikarge S. This is no low risk game: social science researchers reflect on their work. Indian J Med Ethics 2010;7(1):54-5.

28. Rakestraw E. A conflict of interest: why peer review committees need heightened scrutiny under federal antitrust law. J Leg Me 2009;30(4):563-78.

29. Robinson JJ. 'Bad Science' and publication ethics. Int Nurs Rev 2009;56(3):276

30. Sengupta A. Fatal trials: clinical trials are killing people. Indian J Med Ethics 2009;6(3):118-9.

31. Silbergeld E. A question of ethics-publication policy and animals in research. Am J Bioeth 2009;9(12):61-2.

32. Hernández-Ávila M, Garrido-Latorre F, López-Moreno S. Diseño de estudios epidemiológicos. Salud Publica Mex. 2000;42(2):144-54

33. Instituto Mexicano del Seguro Social. Guía para la evaluación de protocolos de investigación. En: Instituto Mexicano del Seguro Social. Procedimiento para la evaluación, registro, seguimiento y modificación de protocolos de investigación en salud presentados ante el Comité Local de Investigación y Ética en Investigación en Salud [monografía en Internet]. México D.F., México: Instituto Mexicano del Seguro Social. 2012 [consultado, abril 2015]. Disponible en http://www.imss. gob.mx/sites/all/statics/profesionalesSalud/investigacionSalud/ normativaInst/2810-003-002_Anexo_4.pdf

34. Presidencia de la República (México). Reglamento de la Ley General de Salud en Materia de Investigación en Salud. México D.F., México: Diario Oficial de la Federación [serie en Internet]. 1982 [consultado, julio 2014]. Disponible en http://www.salud. gob.mx/unidades/cdi/nom/compi/rlgsmis.html 